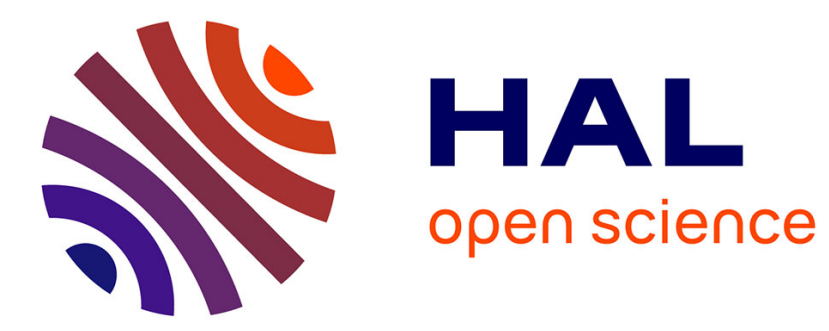

\title{
Stochastic Resource Optimization of Random Access for Transmitters with Correlated Activation
}

Ce Zheng, Malcolm Egan, Laurent Clavier, Anders E Kalør, Petar Popovski

\section{To cite this version:}

Ce Zheng, Malcolm Egan, Laurent Clavier, Anders E Kalør, Petar Popovski. Stochastic Resource Optimization of Random Access for Transmitters with Correlated Activation. IEEE Communications Letters, 2021, 25 (9), pp.3055-3059. 10.1109/LCOMM.2021.3090110 . hal-03212813

\section{HAL Id: hal-03212813 https://hal.science/hal-03212813}

Submitted on 30 Apr 2021

HAL is a multi-disciplinary open access archive for the deposit and dissemination of scientific research documents, whether they are published or not. The documents may come from teaching and research institutions in France or abroad, or from public or private research centers.
L'archive ouverte pluridisciplinaire HAL, est destinée au dépôt et à la diffusion de documents scientifiques de niveau recherche, publiés ou non, émanant des établissements d'enseignement et de recherche français ou étrangers, des laboratoires publics ou privés. 


\title{
Stochastic Resource Optimization of Random Access for Transmitters with Correlated Activation
}

\author{
Ce Zheng, Malcolm Egan, Laurent Clavier, Anders E. Kalør and Petar Popovski
}

\begin{abstract}
For a range of scenarios arising in sensor networks, control and edge computing, communication is event-triggered; that is, in response to the environment of the communicating devices. A key feature of device activity in this setting is correlation, which is particularly relevant for sensing of physical phenomena such as earthquakes or flooding. Such correlation introduces a new challenge in the design of resource allocation and scheduling for random access that aim to maximize throughput or expected sum-rate, which do not admit a closed-form expression. In this paper, we develop stochastic resource optimization algorithms to design a random access scheme that provably converge with probability one to locally optimal solutions of the throughput and the sum-rate. A key feature of the stochastic optimization algorithm is that the number of parameters that need to be estimated grows at most linearly in the number of devices. We show via simulations that our algorithms can outperform existing approaches by up to $30 \%$ for a moderate number of available time slots in realistic networks.
\end{abstract}

Index Terms-random access, stochastic optimization, correlated activation

\section{INTRODUCTION}

A key use case of $5 \mathrm{G}$ communications is machine-type communication, where communications may be event-triggered [1], [2]. That is, transmissions do not occur at periodic intervals, but depend on the environment surrounding a transmitting device with examples arising in sensor networks, control, and in edge computing. As such, event-triggered systems induce a form of random access communications.

An important problem in the design of event-triggered random access networks is the access policy [3], [4], which devices utilize when data is available to transmit. In the context of grant-free access networks, this means that how users select time resources within a frame is governed by a pre-optimized rule.

One approach to optimizing the access policy is to maximize the expected fraction of time slots or subcarriers in which exactly one user transmits - called the throughput in [4]which is relevant when collisions result in the loss of all packets. In the case that devices seek to transmit independently, the resulting policy tends to provide orthogonal resources to devices that are most likely to be active. Several access policies have been proposed including ALOHA [5] and its variants such as coded slotted ALOHA [6], [7], which exploit successive interference cancellation.

C. Zheng (email: chriszhengce123@gmail.com) and L. Clavier (email: laurent.clavier@imt-lille-douai.fr) are with Univ. Lille, CNRS, UMR 8520 - IEMN, and IMT Lille Douai, F-59000 Lille, France. M. Egan (email: malcom.egan@inria.fr) is with Univ Lyon, INSA Lyon, Inria, France. A.E. Kalør (email: aek@es.aau.dk) and P. Popovski (email: petarp@es.aau.dk) are with the Department of Electronic Systems, Aalborg University, Denmark.
However, the design of the access policy is complicated when device activity is correlated. In fact, in the standard formulation of random access, each device is activated independently and randomly. Correlation frequently arises when physical phenomena (such as earthquakes or flooding [8], [9]) are observed. Both the probability that individual devices are active and their correlated activity must be accounted for in resource allocation. As noted in [4], the resulting optimization problem is non-convex and requires a large number of estimated parameters (exponential in the number of devices). For this reason, heuristic time slot allocation algorithms were developed based on upper and lower bounds on the throughput, which involved estimation of a number of parameters that scaled quadratically with the number of devices.

However, a crucial limitation in optimizing access policies based on the throughput, as defined in [4], is the assumption that if a collision occurs then no data can be decoded; despite the fact that multiuser detection strategies can often be employed. In this case, the fading statistics and the spatial distribution of devices also play an important role in determining the system performance. A more appropriate objective is the expected sum-rate. Indeed, maximizing the expected sum-rate increases the likelihood of capture in subsequent successive interference cancellation steps, where the user signals are decoded and cancelled in the order of their signalto-interference-plus-noise ratio [5], [10].

In this paper, we develop time slot allocation algorithms that provably converge to locally optimal solutions for the throughput and expected sum-rate maximization problems and which do not require estimation of a large number of parameters. On the surface, obtaining optimal solutions to these problems appears difficult due to a lack of closed-form expression for the objective, even under simple models such as Rayleigh fading.

We resolve this issue by observing that the expected throughput and expected sum-rate maximization problems are stochastic optimization problems for which stochastic approximation methods [11] can be employed. The resulting low complexity algorithm then only requires realizations of device activity and the signal power of each device. We show via simulation that our throughput optimization algorithm can nearly double the throughput achieved by the algorithms in [4]. Moreover, our algorithm maximizing the expected sum-rate can yield a $30 \%$ improvement of the expected sum-rate over the algorithms in [4] for the key scenario where the number of available time slots is small relative to the number of devices, and the activity of devices is highly correlated. 


\section{SySTEM MODEL}

Consider $N$ devices that aim to transmit data to a common access point utilizing a single common subcarrier. In each frame, a device may transmit within at most one of $K$ time slots, with the possibility that a given device may not seek to transmit any signal. Whether or not a device seeks to transmit in a given frame is modeled probabilistically; i.e., we consider a random access model. To this end, define the device activity random vector $\mathbf{X}=\left(X_{1}, \ldots, X_{N}\right) \in\{0,1\}^{N}$, where device $i$ is active if $X_{i}=1$ and inactive otherwise, with joint probability distribution function $P_{\mathbf{X}}$, which is a multivariate Bernoulli distribution (the most general joint distribution with Bernoulli marginals [12]) without any restriction on parameters. Importantly, the distribution $P_{\mathbf{X}}$ is not known to the access point nor any of the devices nor used in any of the algorithms developed in the sequel.

It is often the case that elements of $\mathbf{X}$, namely $X_{i}, i=$ $1, \ldots, N$, are assumed to be mutually independent; however, in many scenarios dependence naturally arises. For example, consider the case where each device corresponds to a sensor in a network detecting an earthquake. In this setting, devices closest to the epicenter of the earthquake are more likely to detect its presence. As a consequence, we should expect in general that the elements of $\mathbf{X}$ are statistically dependent.

While dependence in device activity complicates the development of statistical models, it also can be exploited to improve system performance. For example, if two devices have highly dependent activity, then they can be allocated different slots within a frame in order to minimize the probability of collision. However, such an allocation is in general non-trivial as it is also necessary to account for the marginal activity distributions. That is, both the dependence and the probability each individual device is active must be accounted for.

Although elements of each activity vector are in general dependent, we assume that a sequence of activity vectors $\mathbf{X}^{1}, \mathbf{X}^{2}, \ldots$ are drawn independently from $P_{\mathbf{X}}$. This scenario can arise when each device is able to transmit data associated with an event within a single slot and events are driven by a stationary process, independent in time.

We consider the transmission protocol given in Algorithm 1. In Step 1, devices randomly select a single slot within the frame according to an allocation matrix $\mathbf{A} \in \mathbb{R}^{N \times K}$, where $A_{i j}$ is the probability that user $i$ transmits in slot $j$ conditioned on activation, and $\sum_{j} A_{i j}=1$. In the sequel, we address how the allocation matrix $\mathbf{A}$ can be optimized.

For each slot $j \in\{1, \ldots, K\}$, detailed in Step 2 for the first slot in Algorithm 1, each device that has selected slot $j$ to transmit their data sends a pilot signal. At this point, as detailed in Step 3, the access point performs multiuser detection-assumed to be error free-in order to detect active devices in slot $j$ and their corresponding channel gains for the common subcarrier utilized by all devices, denoted by $\left\{\left|g_{i}\right|^{2}\right\}_{i \in \mathcal{S}_{j}}$, where $\mathcal{S}_{j}$ is the set of active devices in slot $j$.

To perform data decoding, the access point exploits successive interference cancellation [13]. In more detail, suppose that each slot $j$ consists of $T$ channel uses and the received

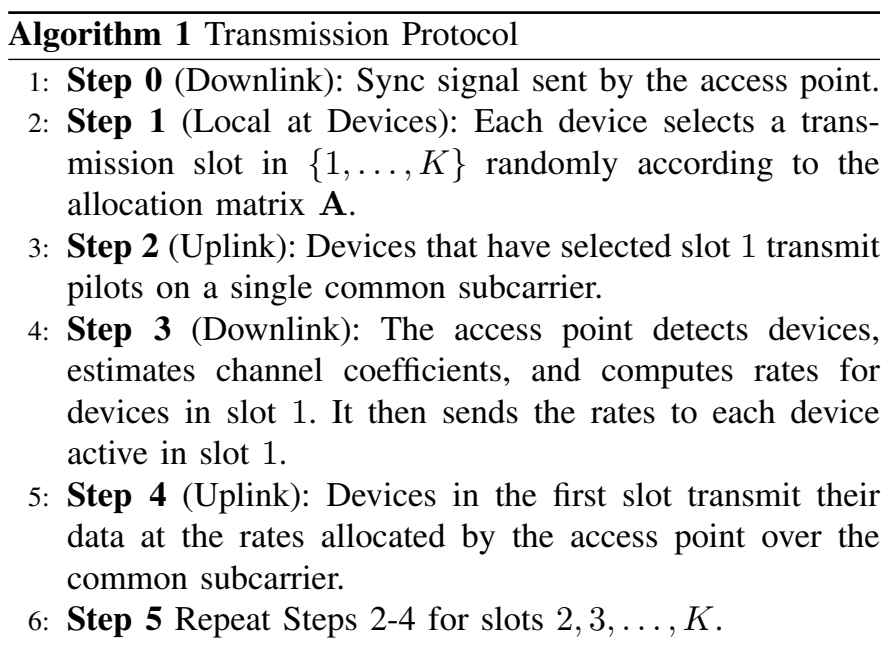

signal on the common subcarrier is given by

$$
\mathbf{y}_{j}=\mathbf{D}_{j} \mathbf{g}+\mathbf{w}_{j}
$$

where $\mathbf{D}_{j} \in \mathbb{C}^{T \times N}$ is the data to be transmitted by all devices. The coefficients $g_{i}=\sqrt{P_{i}} r_{i}^{-\eta / 2} h_{i}, i \in\{1, \ldots, N\}$ with $h_{i} \in$ $\mathbb{C}, P_{i}, r_{i}^{-\eta / 2}$ and $\eta$ denoting the block fading coefficient, fixed transmit power, path loss and path loss exponent for the $i$-th device, respectively, assumed to be constant for each device over the $T$ channel uses. For $i \notin \mathcal{S}_{j}$, we set the $i$-th column of $\mathbf{D}_{j}$ to be zero. The term $\mathbf{w}_{j} \sim \mathcal{C N}\left(0, \sigma^{2} \mathbf{I}_{T \times T}\right)$ is additive white Gaussian noise.

Under successive interference cancellation and sufficiently large $T$, the achievable rate for device $m \in \mathcal{S}_{j}$ with the $m$-th largest channel gain in $\left\{\left|g_{i}\right|^{2}\right\}_{i \in \mathcal{S}_{j}}$, denoted by $\left|g_{m}\right|^{2}$, is well approximated by

$$
R_{m}=W \log \left(1+\frac{\left|g_{m}\right|^{2}}{\sigma^{2}+\sum_{m<l \leq\left|\mathcal{S}_{j}\right|}\left|g_{l}\right|^{2}}\right), m=1, \ldots,\left|\mathcal{S}_{j}\right|
$$

where $W$ is available bandwidth and assumed in the sequel, without loss of generality, to be $W=1$. As such, $R_{m}$ is the rate sent to the device in $\mathcal{S}_{j}$ with the $m$-th largest channel gain, as detailed at the end of Step 3 for the first slot. We note that in practice, the rate may be quantized in order to limit the amount of feedback that is necessary.

Finally, in Step 4 for devices in slot 1, data transmission occurs for the devices that are active in slot $j$. In particular, each device utilizes a rate given by (2).

A key feature of the protocol in Algorithm 1 is that downlink transmissions occur for each slot within the frame in order to inform devices of their rate. While increasing the required feedback, it allows for devices to send data using a rate tailored to their channel instead of at a fixed rate. While not useful for cheap sensors transmitting very small quantities of data, it is highly desirable for high-resolution data such as arising from video cameras and LIDAR.

Another feature of Algorithm 1 is that the access point is not required to detect all active devices at the beginning of the frame. Instead, detection is done on a slot-by-slot basis. As such, a smaller quantity of devices need to be detected 
within each slot, which reduces the difficulty of the practically important problem of multiuser detection.

\section{OPTIMIZATION CRITERIA}

The key question addressed in this paper is how to optimize the allocation matrix $\mathbf{A}$, assigning a probability for each active device to access each slot. One natural criterion is the expected fraction of slots in which exactly one user transmits, or the expected throughput, which was considered in [4]. Formally, the expected throughput is given by

$$
T_{1}(\mathbf{A})=\mathbb{E}_{\mathbf{X}}\left[T_{1}^{\mathbf{X}}(\mathbf{A})\right]
$$

where

$$
T_{1}^{\mathbf{X}}(\mathbf{A})=\sum_{n=1}^{N} \sum_{k=1}^{K} X_{n} A_{n k} \prod_{\substack{m=1 \\ m \neq n}}^{N}\left(1-X_{m} A_{m k}\right),
$$

which follows from an analysis of the probability a single device transmits in each time slot $k$.

An alternative criterion is to maximize the expected sumrate, accounting for successive interference cancellation. In particular, the expected sum-rate is given by

$$
T_{2}(\mathbf{A})=\mathbb{E}_{\mathbf{h}, \mathbf{X}}\left[T_{2}^{\mathbf{h}, \mathbf{X}}(\mathbf{A})\right]
$$

where

$$
\begin{aligned}
T_{2}^{\mathbf{h}, \mathbf{X}}(\mathbf{A})= & \sum_{k=1}^{K} \sum_{\mathcal{S} \in \mathcal{P}(N)} Q_{k}(\mathcal{S} \mid \mathbf{X}) \\
& \cdot \sum_{m=1}^{|\mathcal{S}|} \log \left(1+\frac{\left|g_{\mathcal{S}(m)}\right|^{2}}{\sigma^{2}+\sum_{m<l \leq|\mathcal{S}|}\left|g_{\mathcal{S}(l)}\right|^{2}}\right)
\end{aligned}
$$

with

$$
Q_{k}(\mathcal{S} \mid \mathbf{X})=\prod_{i \in \mathcal{S}} X_{i} A_{i k} \prod_{j \in \mathcal{S}^{c}}\left(1-X_{j} A_{j k}\right),
$$

which corresponds to the probability the devices in $\mathcal{S}$ transmit in slot $k$ conditioned on the activity vector $\mathbf{X}$. Under the protocol in Algorithm 1, the expected sum-rate corresponds to the total sum-rate for each frame averaged over user activity and channel coefficients.

The criteria in (3) and (5) do not in general admit closedform expressions - partly due to the fact that $P_{\mathbf{X}}$ is not known to the access point-and are non-convex functions of the continuous variables in $\mathbf{A}$. In the following section, we propose new algorithms for the optimization of the allocation matrix A with respect to these criteria, with provable convergence guarantees. In particular, we seek solutions

$$
\mathbf{A}_{1}^{*}=\arg \max _{\substack{\mathbf{A} \in \mathbb{R}_{+}^{N \times K}: \\ \sum_{j} A_{i j}=1, i=1, \ldots, N}} T_{1}(\mathbf{A})
$$

and

$$
\mathbf{A}_{2}^{*}=\arg \max _{\substack{\mathbf{A} \in \mathbb{R}_{+}^{N \times K}: \\ \sum_{j} A_{i j}=1, i=1, \ldots, N}} T_{2}(\mathbf{A}) .
$$

At present, only solutions based on tractable bounds of (3) have been obtained for the problem (8) in [4]. In contrast, our algorithms directly solve the problems in (8) and (9) and provably converge with probability one to a local maximum.

\section{Optimization of the Allocation Matrix}

A key observation is that the optimization problems in (8) and (9) are stochastic optimization problems with $P_{\mathbf{X}}$ unknown. As a consequence, (8) and (9) can be solved via stochastic approximation algorithms [11], which exploit samples from $P_{\mathbf{X}}$. In particular, since the functions inside the expectations arising in (3) and (5) are continuously differentiable in $\mathbf{A}$, stochastic gradient ascent (SGA) can be applied. We note that as $P_{\mathbf{X}}$ is unknown, gradient ascent methodswhere the gradient is available - cannot be applied since even the objective cannot be evaluated. Moreover, as we will show, the algorithm converges to a locally optimal solution with probability one.

In this section, we derive the SGA algorithms for the problems in (8) and (9). Suppose that for a sequence of training frames, devices access slots according to an initial allocation matrix $\mathbf{A}^{1}$. The access point can then obtain knowledge of device activity via multiuser detection; namely, in each frame $t$, the activity vector $\mathbf{X}^{t}, t=1,2, \ldots$. Note that data can be transmitted during the training frames, as the same multiuser detection algorithm is required for data decoding.

\section{A. SGA Algorithm for (8)}

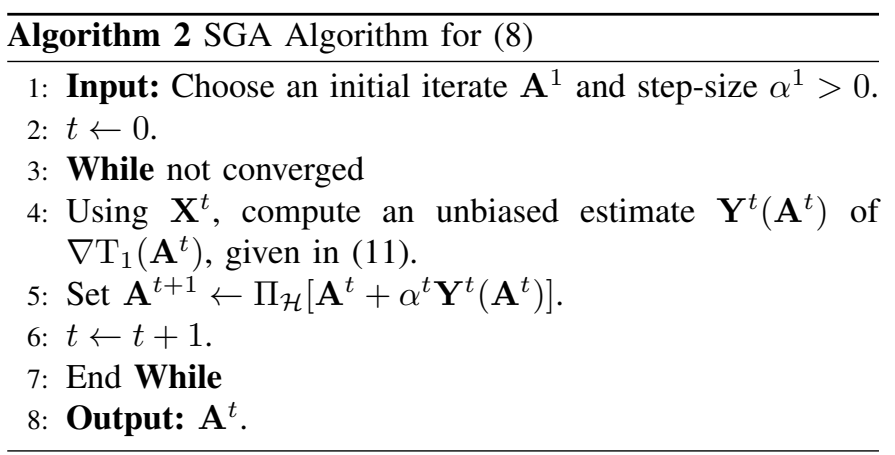

Algorithm 2 provides details of the throughput maximization algorithm to solve $(8)$, where $\Pi_{\mathcal{H}}[\cdot]$ denotes the closest point (w.r.t. $\|\cdot\|_{F}$ ) in the constraint set $\mathcal{H}=\left\{\mathbf{A} \in \mathbb{R}_{+}^{N \times K}\right.$ : $\left.\sum_{j} A_{i j}=1, i=1, \ldots, N\right\}$ from $\mathbf{A}$. The term $\mathbf{Y}^{t}\left(\mathbf{A}^{t}\right)$ is an unbiased estimate of the gradient of the throughput $T_{1}$ at $\mathbf{A}^{t}$. To compute the estimate, first observe that

$$
\begin{aligned}
& \frac{\partial T_{1}}{\partial A_{q l}}(\mathbf{A}) \\
= & \mathbb{E}_{\mathbf{X}}\left[X_{\substack{m=1 \\
m \neq q}}^{N}\left(1-X_{m} A_{m l}\right)-\sum_{\substack{n=1 \\
n \neq q}}^{N} X_{q} X_{n} A_{n l} \prod_{\substack{m=1 \\
m \neq n \\
m \neq q}}^{N}\left(1-X_{m} A_{m l}\right)\right.
\end{aligned} .
$$

Given a realization $\mathbf{X}^{i}$, the unbiased estimate of the gradient in the $t$-th iteration is then given by

$$
Y_{q l}^{t}(\mathbf{A})=X_{q}^{t} \prod_{\substack{m=1 \\ m \neq q}}^{N}\left(1-X_{m}^{t} A_{m l}\right)-\sum_{\substack{n=1 \\ n \neq q}}^{N} X_{q}^{t} X_{n}^{t} A_{n l} \prod_{\substack{m=1 \\ m \neq n \\ m \neq q}}^{N}\left(1-X_{m}^{t} A_{m l}\right)
$$


Under certain conditions on the step-size sequence $\left\{\alpha^{t}\right\}$, Algorithm 2 converges with probability one to a local maximum. We establish this result in the following Theorem 1.

Theorem 1. Suppose that the step-size sequence $\left\{\alpha^{t}\right\}$ satisfies

(i) $\sum_{t=1}^{\infty}\left(\alpha^{t}\right)^{2}<\infty$;

(ii) $\sum_{t=1}^{\infty} \alpha^{t}=\infty, \alpha^{t} \geq 0, t \geq 1 ; \alpha^{t}=0, t<1$.

Then, the iterates $\mathbf{A}^{t}$ in Algorithm 2 converge almost surely as $t \rightarrow \infty$ to a local maximum of the problem (8).

Proof. The proof of Theorem 1 involves verifying Assumptions (A6.1.1)-(A6.1.7) and (A4.3.3) in [11] and applying [11, Theorem 6.1.1]. Due to the fact that (11) is bounded and Lipschitz continuous in A, (A6.1.1)-(A6.1.7) follow immediately. Assumption (A4.3.3) holds since the constraint set $\mathcal{H}$ forms a linear connected compact surface and hence has a continuously differentiable outer normal.

\section{B. SGA Algorithm for (9)}

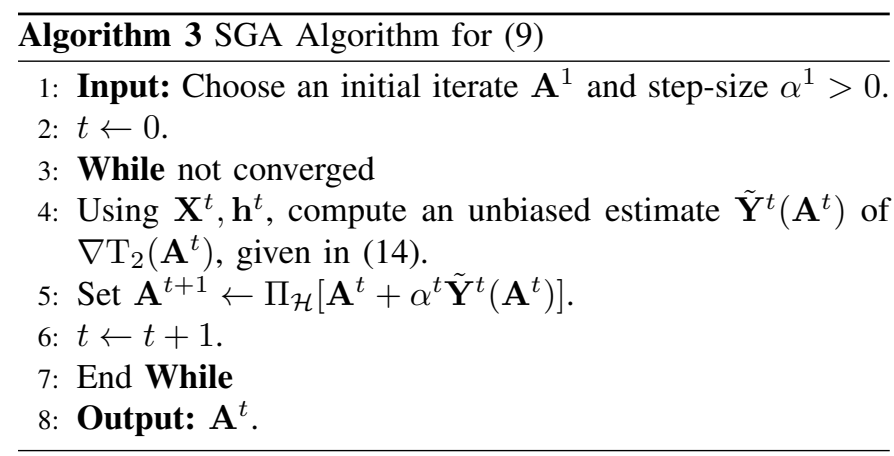

We now turn to the expected sum-rate maximization problem in (5), for which the allocation $\mathbf{A}$ is obtained via Algorithm 3 . In this case, we have

$$
\begin{gathered}
\frac{\partial T_{2}}{\partial A_{q l}}(\mathbf{A})=\mathbb{E}_{\mathbf{X}, \mathbf{h}}\left[\sum_{\mathcal{S} \in \mathcal{P}(N)} \frac{\partial Q_{q}(\mathcal{S} \mid \mathbf{X})}{\partial A_{q l}}\right. \\
\left.\quad \cdot \sum_{m=1}^{|\mathcal{S}|} \log \left(1+\frac{\left|g_{\mathcal{S}(m)}\right|^{2}}{\sigma^{2}+\sum_{m<p \leq|\mathcal{S}|}\left|g_{\mathcal{S}(p)}\right|^{2}}\right)\right],
\end{gathered}
$$

where

$$
\begin{aligned}
& \frac{\partial Q_{q}(\mathcal{S} \mid \mathbf{X})}{\partial A_{q l}} \\
& =\left\{\begin{array}{l}
X_{q} \prod_{i \in \mathcal{S} \backslash\{q\}} X_{i} A_{i l} \prod_{j \in \mathcal{S}^{c}}\left(1-X_{j} A_{j l}\right), \\
-X_{q} \prod_{i \in \mathcal{S}} X_{i} A_{i l} \prod_{j \in \mathcal{S}^{c} \backslash\{q\}}\left(1-X_{j} A_{j l}\right), \quad q \in \mathcal{S}
\end{array}\right.
\end{aligned}
$$

Given realizations of $\mathbf{X}^{t}$ and $\mathbf{h}^{t}$, an unbiased estimate of the gradient in the $t$-th iteration is then given by

$$
\begin{aligned}
\tilde{Y}_{q l}^{t}(\mathbf{A}) & =\sum_{\mathcal{S} \in \mathcal{P}(N)} \frac{\partial Q_{q}\left(\mathcal{S} \mid \mathbf{X}^{t}\right)}{\partial A_{q l}} \\
& \cdot \sum_{m=1}^{|\mathcal{S}|} \log \left(1+\frac{\left|g_{\mathcal{S}(m)}^{t}\right|^{2}}{\sigma^{2}+\sum_{m<p \leq|\mathcal{S}|}\left|g_{\mathcal{S}(p)}^{t}\right|^{2}}\right) .
\end{aligned}
$$

Algorithm 3 then has the following convergence behavior, which can be proved using the same argument as for Theorem 1 .

Theorem 2. Suppose that the step-size sequence $\left\{\alpha^{t}\right\}$ satisfies

(i) $\sum_{t=1}^{\infty}\left(\alpha^{t}\right)^{2}<\infty$;

(ii) $\sum_{t=1}^{\infty} \alpha^{t}=\infty, \alpha^{t} \geq 0, t \geq 1 ; \alpha^{t}=0, t<1$.

Then, the iterates $\mathbf{A}^{t}$ in Algorithm 3 converge almost surely as $t \rightarrow \infty$ to a local maximum of the problem (9).

\section{Simulation Results}

To evaluate our algorithm, we consider the following eventtriggered scenario based on the model introduced in [4]. In particular, events are generated according to a homogeneous Poisson point process on $[0, L]^{2}$ with rate $\lambda$ and a given device transmits if an event occurs within a radius $R$ of the device. Throughout our numerical study, we assume that $L=50$, $\lambda=10 /(L+2 R)^{2}$ and $\sigma^{2}=1$. In this model, each device has the same activity probability given by

$$
\mathbf{E}\left[X_{i}\right]=1-\exp \left\{-\lambda \pi R^{2}\right\} .
$$

We first compare Algorithm 2 for throughput optimization with the algorithm proposed in [4], called Algupper and

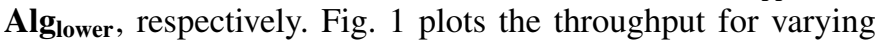
numbers of slots $K$ with $N=50$ devices in the network. For Algorithm 2, we consider two choices for the initial value of the allocation $\mathbf{A}^{1}$ :

$$
\mathbf{A}_{0}=\left[\begin{array}{cccc}
1 & 0 & \ldots & 0 \\
1 & 0 & \ldots & 0 \\
\vdots & \vdots & \ddots & 0 \\
1 & 0 & & 0
\end{array}\right]_{N \times K}
$$

and $\mathbf{A}_{1}$, which corresponds to the allocation obtained via Algupper. To compute the allocation, 100 iterations of Alg. 2 were used with step-size sequence $\alpha^{k}=10 / k$.

Observe in Fig. 1 that for 10 slots, corresponding to $20 \%$ of the total number of devices, the throughput for Algorithm 2 is approximately double that of the algorithms in [4] with the choice $\mathbf{A}^{1}=\mathbf{A}_{0}$. It is only for large numbers of available slots that $\mathbf{A}_{1}$ outperforms the algorithms in [4]. This suggests that performance is sensitive to the initial allocation due to the presence of poor local maxima.

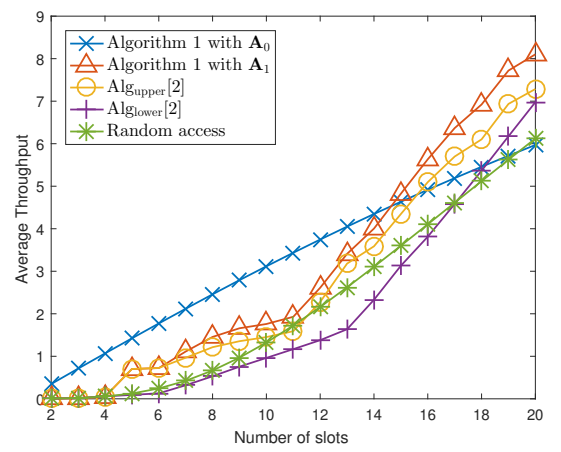

Fig. 1. Throughput with $N=50$ for varying $K$ with $\lambda=10 / 80^{2}$. 
We now turn to evaluate the algorithms in terms of the expected sum-rate. To do so, we assume Rayleigh fading, $\left|h_{i}\right|^{2} \sim \exp (1)$, and a path-loss exponent $\eta=3$.

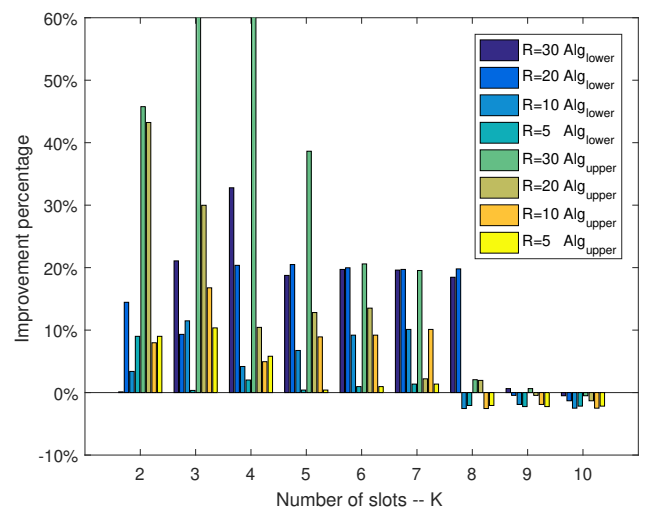

Fig. 2. Improvement of SGA over $\mathbf{A l g}_{\text {lower }}$ and $\mathbf{A l g u p p e r ~ [ 4 ] ~ w i t h ~} N=16$ for varying $K$ with $\mathbf{A}^{1}=\mathbf{A}_{0}$, where $\lambda=10 /(50+2 R)^{2}$.

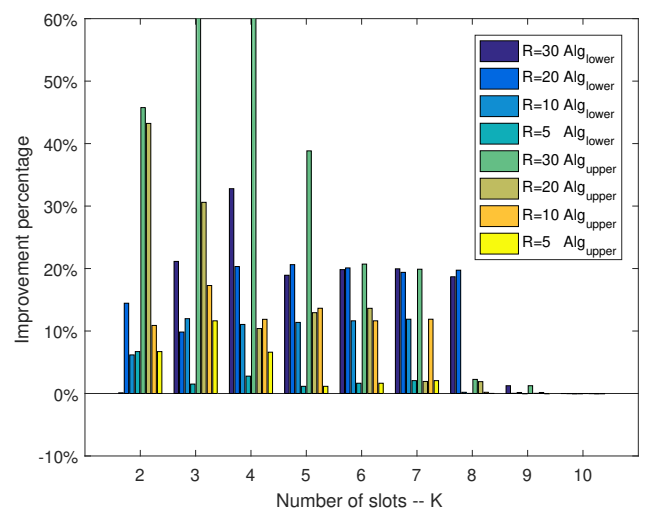

Fig. 3. Improvement of SGA over $\mathbf{A l g}_{\text {lower }}$ and $\mathbf{A l g} \operatorname{lgpper}_{\text {[4] with } N=16}$ for varying $K$ with $\mathbf{A}^{1}=\mathbf{A}_{1}$, where $\lambda=10 /(50+2 R)^{2}$.

Fig. 2 and Fig. 3 plot the percentage improvement in the expected sum-rate of Algorithm 3 over the methods in [4] with $N=16$ devices in the network located at

$$
\begin{aligned}
& \mathbf{d} \\
= & {[0.429,0.623,1.052,1.814,2.703,4.684,5.868,6.374,} \\
& 6.791,6.839,7.142,8.126,8.408,8.802,8.963,9.758] .
\end{aligned}
$$

These device locations are chosen to illustrate the impact of a small number of devices close to the access point while the others are distributed further away, which is the case where there are several strong interferers in the system. To compute the allocation in each case, 100 iterations of Alg. 3 were used with step-size sequence $\alpha^{k}=10 / k$.

As for the throughput, there are significant gains for moderate numbers of available slots. This is particularly clear in Fig. 3 with initial allocation $\mathbf{A}_{1}$ when the event radius is $R=30$ (corresponding to high correlation). In this case, Alg. 3 yields an improvement of over $30 \%$. This is largely due to the fact that Algorithm 3 accounts for both correlation and the location of devices, unlike the algorithms in [4] where only correlation is accounted for. For all choices of $R$, the initial allocation $\mathbf{A}^{1}=\mathbf{A}_{1}$ outperforms the algorithms in [4]. With $\mathbf{A}^{1}=\mathbf{A}_{0}$, Alg. 3 has a lower performance for most values of $R$, suggesting that knowledge of the correlations required for $\mathbf{A l g}_{\text {upper }}$ are particularly useful in expected sumrate maximization.

\section{CONCLUSIONS}

A key problem is designing resource allocation in eventtriggered random access systems is coping with the stochastic nature of device activity, which is complicated further in the presence of correlation. In this paper, we developed low complexity algorithms based on stochastic optimization in order to maximize the throughput and the expected sum-rate, which both provably converge to a local maxima with probability one. A feature of our approach is that the parameters of the activity distribution, exponential in the number of devices, do not have to be estimated separately as in standard gradient ascent methods. Our algorithms are also shown via simulations to significantly outperform the state-of-the-art methods in [4], while also avoiding the need to estimate a large number of parameters.

\section{ACKNOWLEDGEMENTS}

This work has been (partly) funded by the French National Agency for Research (ANR) under grant ANR-16-CE25-0001 - ARBURST and the COST Action CA15104 IRACON.

\section{REFERENCES}

[1] J. Navarro-Ortiz et al., "A survey on 5G usage scenarios and traffic models," IEEE Communications Surveys \& Tutorials, vol. 22, no. 2, pp. 905-929, 2020.

[2] F. Metzger, T. Hoßfeld, A. Bauer, S. Kounev, and P. Heegaard, "Modeling of aggregated iot traffic and its application to an iot cloud," Proceedings of the IEEE, vol. 107, pp. 679-694, Apr. 2019.

[3] M. Emara, H. ElSawy, and G. Bauch, "A spatiotemporal model for peak aoi in uplink iot networks: Time versus event-triggered traffic," IEEE Internet of Things Journal, vol. 7, no. 8, pp. 6762-6777, 2020.

[4] A. E. Kalør, O. A. Hanna, and P. Popovski, "Random access schemes in wireless systems with correlated user activity," in 2018 IEEE 19th International Workshop on Signal Processing Advances in Wireless Communications (SPAWC), pp. 1-5, IEEE, 2018.

[5] L. Roberts, "Aloha packet system with and without slots and capture," SIGCOMM Comput. Commun. Rev., vol. 5, pp. 28-42, Apr. 1975.

[6] E. Paolini, G. Liva, and M. Chiani, "Coded slotted aloha: a graphbased method for uncoordinated massive access," IEEE Transactions on Information Theory, vol. 61, pp. 6815-6832, Dec. 2015.

[7] C. Stefanovic and P. Popovski, "Aloha random access that operates as a rateless code," IEEE Transactions on Communications, vol. 61, pp. 4653-4662, Nov. 2013.

[8] G. Werner-Allen et al., "Monitoring volcanic eruptions with a wireless sensor network," in Proc. of the Second European Workshop on Wireless Sensor Networks, 2005.

[9] M. Meyer et al., "Event-triggered natural hazard monitoring with convolutional neural networks on the edge," in Proc. ACM/IEEE International Conference on Information Processing in Sensor Networks (IPSN), 2019.

[10] L. Dai et al., "Non-orthogonal multiple access for 5G: solutions, challenges, opportunities, and future research trends," IEEE Communications Magazine, vol. 53, no. 9, pp. 74-81, 2015.

[11] H. Kushner and G. Yin, Stochastic approximation and recursive algorithms and applications. Springer Science \& Business Media, 2003.

[12] B. Dai, S. Ding, and G. Wahba, "Multivariate Bernoulli distribution," Bernoulli, vol. 19, no. 4, pp. 1465-1483, 2013.

[13] S. Verdú, Multiuser Detection. New York: Cambridge University Press, 1998. 